valves were involved in 9 and 8 cases, respectively. Cardiac involvement was silent in $36.4 \%$ and occurred as an initial presenting symptom of SLE in $9.1 \%$ cases. Biologically, patients with cardiac involvement had higher levels of CRP and anti dsDNA, and lower levels of complement C3 compared to patients with no cardiac involvement; while SLE activity was the only significant predictor for cardiac involvement $($ Beta $=0.654 ; \mathrm{p}=0.020)$

Conclusions Cardiac complications are common (1 out of 3 times). They are predicted by high SLE activity and antidsDNA, CRP and low C3 levels. Reguiar echocardiography is erommended for Patients with high SLE activity.

\section{OUTCOMES OF 847 CHILDHOOD-ONSET SYSTEMIC LUPUS ERYTHEMATOUSUS PATIENTS IN THREE AGE GROUPS}

${ }^{1} \mathrm{~L}$ Campos*, ${ }^{2} \mathrm{~S}$ Lopes, ${ }^{2} \mathrm{~N}$ Gormezano, ${ }^{3} \mathrm{R}$ Gomes, ${ }^{2} \mathrm{~N}$ Aikawa, ${ }^{2} \mathrm{R}$ Pereira, ${ }^{4} \mathrm{M}$ Terreri, ${ }^{5} \mathrm{C}$ Magalhaes, ${ }^{6} \mathrm{E}$ Okuda, ${ }^{4} \mathrm{~A}$ Sakamoto, ${ }^{3} \mathrm{~A}$ Sallum, ${ }^{7} \mathrm{~S}$ Appenzeller, ${ }^{8} \mathrm{~V}$ Ferriani, ${ }^{9} \mathrm{C}$ Barbosa, ${ }^{10} \mathrm{~S}$ Lotufo, ${ }^{4} \mathrm{~L}$ Andrade, ${ }^{2} \mathrm{E}$ Bonfa, ${ }^{3} \mathrm{C}$ Silva. ${ }^{1}$ SAO PAULO, Brazil, ${ }^{2}$ Faculdade de Medicina da Universidade de São Paulo, Division of Rheumatology, Sao Paulo, Brazil; ${ }^{3}$ Children's Institute- Faculdade de Medicina da Universidade de São Paulo, Paediatric Rheumatology Unit, Sao Paulo, Brazil; ${ }^{4}$ Universidade Federal de São Paulo, Paediatric Rheumatology Unit, Sao Paulo, Brazil; ${ }^{5}$ São Paulo State University UNESP - Faculdade de Medicina de Botucatu, Paediatric Rheumatology Division, Sao Paulo, Brazil; ${ }^{6}$ Irmandade da Santa Casa de Misericórdia de São Paulo, Paediatric Rheumatology Unit, Sao Paulo, Brazil ; ' State University of Campinas UNICAMP, Paediatric Rheumatology Unit, Sao Paulo, Brazil; ${ }^{8}$ Ribeirão Preto Medical School - University of São Paulo, Paediatric Rheumatology Unit, Sao Paulo, Brazil; ${ }^{9}$ Hospital Infantil Darcy Vargas, Paediatric Rheumatology Unit, Sao Paulo, Brazil; ${ }^{10}$ Hospital Menino Jesus, Paediatric Rheumatology Unit, Sao Paulo, Brazil

\subsection{6/lupus-2017-000215.128}

Background and aims To assess outcomes of childhood systemic lupus erythematosus (cSLE) in three different age groups evaluated at last visit: group A early-onset disease( $<6$ years), group $B$ school age $(\geq 6$ and $<12$ years $)$ and group $C$ adolescent ( $\geq 12$ and $<18$ years).

Methods Observational cohort study of 10 Paediatric Rheumatology centres, including 847 cSLE patients.

Results Group A had 39 (4\%), B 395 (47\%) and C 413 (49\%). Median disease duration was higher in group A compared to groups B and $\mathrm{C}[8.3(0.1-23.4)$ vs. $6.2(0-17)$ vs. 3.3 $(0-14.6)$ years, $\mathrm{p}<0.0001]$. The median SLICC/ACR-DI[0 (0$9)$ vs. $0(0-6)$ vs. $0(0-7), p=0.065]$ was comparable in all groups. Further analysis of organ/system damage revealed that frequencies of neuropsychiatric(21\% vs. $10 \%$ vs. $7 \%$, $\mathrm{p}=0.007)$, skin $(10 \%$ vs. $1 \%$ vs. $3 \%, \mathrm{p}=0.002)$ and peripheral vascular involvements(5\% vs. $3 \%$ vs. $0.3 \%, p=0.008)$ were more frequent in group A compared to B and C. Frequencies of severe cumulative lupus manifestations such as nephritis, thrombocytopenia and autoimmune hemolytic anaemia were similar in all groups $(\mathrm{p}>0.05)$. Mortality rate was higher in group A compared to groups B and C(15\% vs. $10 \%$ vs. $6 \%$, $\mathrm{p}=0.028)$. Out of 69 deaths, 33/69 (48\%) occurred within the first two years after diagnosis. Infections accounted for $54 / 69(78 \%)$ of the deaths and 38/54 (70\%) had concomitant disease activity.

Conclusions: This large multicenter study provided evidence that early-onset cSLE group had distinct outcomes, with higher mortality rate and neuropsychiatric/vascular/skin organ damages in spite of comparable frequencies of severe cumulative lupus manifestations. We also identified that overall death in cSLE patients was an early event mainly attributed to infection associated with disease activity.

\section{9 PANNICULITIS IN CHILDHOOD-ONSET SYSTEMIC LUPUS ERYTHEMATOSUS: A MULTICENTRIC COHORT STUDY}

'L Campos*, ${ }^{2} \mathrm{M}$ Verdier, ${ }^{2} \mathrm{P}$ Anuardo, ${ }^{2} \mathrm{~N}$ Gormezano, ${ }^{3} \mathrm{R}$ Romiti, ${ }^{4} \mathrm{~N}$ Aikawa, ${ }^{4} \mathrm{R}$ Pereira, ${ }^{5} \mathrm{M}$ Terreri, ${ }^{6} \mathrm{C}$ Magalhaes, ${ }^{2} \mathrm{~J}$ Ferreira, ${ }^{2} \mathrm{M}$ Silva, ${ }^{2} \mathrm{M}$ Ferriani, ${ }^{5} \mathrm{~A}$ Sakamoto, ${ }^{7} \mathrm{~V}$ Ferriani, ${ }^{8} \mathrm{M}$ Centeville, ${ }^{6} \mathrm{~J}$ Sato, ${ }^{9} \mathrm{M}$ Santos, ${ }^{4} \mathrm{E}$ Bonfa, ${ }^{2} \mathrm{C}$ Silva. ${ }^{1} \mathrm{SAO}$ PAULO, Brazil; ${ }^{2}$ Children's Institute- Faculdade de Medicina da Universidade de São Paulo, Paediatric Rheumatology Unit, Sao Paulo, Brazil; ${ }^{3}$ Faculdade de Medicina da Universidade de São Paulo, Division of Dermatology, Sao Paulo, Brazil; ${ }^{4}$ Faculdade de Medicina da Universidade de São Paulo, Division of Rheumatology, Sao Paulo, Brazil; ${ }^{5}$ Universidade Federal de São Paulo, Paediatric Rheumatology Unit, Sao Paulo, Brazil; ${ }^{6}$ São Paulo State University UNESP - Faculdade de Medicina de Botucatu, Paediatric Rheumatology Unit, Sao Paulo, Brazil; ${ }^{7}$ Ribeirão Preto Medical School - University of São Paulo, Paediatric Rheumatology Unit, Sao Paulo, Brazil; ${ }^{8}$ São Paulo State University of Campinas UNICAMP, Paediatric Rheumatology Unit, Sao Paulo, Brazil; ${ }^{9}$ Irmandade da Santa Casa de Misericórdia de São Paulo, Paediatric Department, Sao Paulo, Brazil

\subsection{6/lupus-2017-000215.129}

Background and Aims To evaluate prevalence, clinical manifestations, laboratory abnormalities, treatment and outcome in a multicenter cohort of childhood-onset systemic lupus erythematosus(cSLE) patients with and without panniculitis.

Methods Panniculitis was diagnosed due to painful subcutaneous nodules and/or plaques in deep dermis/subcutaneous tissues and lobular/mixed panniculitis with lymphocytic lobular inflammatory infiltrate in skin biopsy. Statistical analysis was performed using Bonferroni correction $(\mathrm{p}<0.004)$.

Results Panniculitis was observed in 6/847 (0.7\%) cSLE. Painful subcutaneous erythematosus and indurated nodules were observed in 6/6 panniculitis patients and painful subcutaneous plaques in $4 / 6$. Generalised distribution was evidenced in $3 / 6$ and localised in upper limbs in $2 / 6$ and face in 1/6. Histopathology features showed lobular panniculitis without vasculitis in $5 / 6$ (one of them had concomitant obliterative vasculopathy due to antiphospholipid syndrome) and panniculitis with vasculitis in $1 / 6$. Comparison between cSLE with panniculitis and 60 cSLE without panniculitis with same disease duration [2.75 $(0-11.4)$ vs. $2.83(0-11.8)$ years, $\mathrm{p}=0.297]$, showed higher frequencies of constitutional involvement (67\% vs. $10 \%$, $\mathrm{p}=0.003)$, leukopenia (67\% vs. $7 \%, \mathrm{p}=0.002)$ and median Creactive protein $(10.5$ vs. $0.5 \mathrm{mg} / \mathrm{L}, \mathrm{p}=0.001)$. Cutaneous atrophy and hyperpigmentation occurred in $83 \%$ of patients.

Conclusions Panniculitis is a rare skin manifestation of cSLE occurring in the first three years of disease with considerable sequelae. The majority of patients have concomitant mild lupus manifestations.

\section{NORMAL-PRESSURE HYDROCEPHALUS IN A NINE YEAR OLD FILIPINO FEMALE WITH SYSTEMIC LUPUS ERYTHEMATOSUS}

MT Collante*, C Bernal. University of Santo Tomas Hospital, Department of PediatricsSection of Paediatric Rheumatology, Manila, Philippines

\subsection{6/lupus-2017-000215.130}

Background and Aims Central nervous system involvement in paediatric systemic lupus erythematosus (SLE) is common, however, normal-pressure hydrocephalus is unusual.

Methods The medical records of a nine-year old Filipino female with SLE was reviewed.

Results Patient presented with prolonged fever, weight loss, generalised weakness, lapses in memory, and mood lability. She was poorly nourished, non-ambulatory, pale, febrile, and ill-looking. She was tachycardic, with normal blood pressure, 
decreased breath sounds over lung bases, a systolic murmur over the apex, and significant contractures on elbows and knees. She was coherent, oriented to all spheres, with intact cranial nerves, but with impaired recall. Upper extremity muscle testing was $4 / 5$, while that of the lower extremities were $3 / 5$. There was severe muscle atrophy, no fasciculations or other involuntary movements, no sensory deficits, no abnormal reflexes, and no signs of meningeal irritation. No oral ulcers, joint swelling, nor rash was noted. Work up showed anaemia, leukopenia, positive ANA, and positive antidsDNA. Serum electrolytes and urinalysis were normal. Chest radiograph showed interstitial infiltrates, while Mantoux test was negative. Cranial magnetic resonance imaging with contrast showed abnormal white matter intensities at the frontoparietal, periventricular, and subcortical regions, communicating hydrocephalus, and cortical cerebral and cerebellar atrophy. Cerebrospinal fluid analysis was normal. She showed remarkable improvement with intravenous antibiotics and corticosteroids. Upon discharge, she had good well-being, appetite, cognition, and mood, and was already able to ambulate with assistance. She received cyclophosphamide pulse therapy and underwent physical rehabilitation.

Conclusions Normal-pressure hydrocephalus may be part of the presenting manifestations of paediatric SLE.

\section{OUTCOME OF FILIPINO CHILDREN WITH LUPUS NEPHRITIS TREATED WITH A MODIFIED TREATMENT REGIMEN USING CYCLOPHOSPHAMIDE}

MT Collante*, C Bernal. University of Santo Tomas Hospital, Department of PediatricsSection of Paediatric Rheumatology, Manila, Philippines

\subsection{6/lupus-2017-000215.131}

Background and Aims The current therapeutic strategy for childhood-onset lupus nephritis (LN) involves an induction phase, aiming to promote remission, and a maintenance phase control disease and prevent relapses. Various regimens have been used worldwide, which differ in drug of choice and dosage, and duration of the induction and maintenance phases. This study evaluated treatment outcome and adverse event occurrence in Filipinos with childhood-onset LN.

Methods Medical records of patients diagnosed with childhood-onset LN who received an extended induction phase of 9 months followed by a maintenance phase of 5 quarterly intravenous cyclophosphamide pulses from year 2006 to 2014 at the University of Santo Tomas Hospital were reviewed.

Results Nineteen patients completed the modified regimen $\mathbf{9 4 . 7 \%}$ female, mean age $11.2+3.7$ years at lupus diagnosis, mean LN duration to completion of treatment $30.6+5.2$ months). At 9 months, 47.4\% (9/19) reached complete remission, and $52.6 \%(10 / 9)$ were in partial remission. After 9 monthly and 5 quarterly pulses, 94.7\% (18/19) was with complete treatment response. One patient relapsed during the maintenance phase and was with partial response at the end of treatment. The random urine protein:creatinine ratio and disease activity were significantly improved in all 19 patients. Treatment failure was not noted in any of the patients at the end of maintenance phase and at completion. Reported adverse events were gastrointestinal symptoms (100\%), mild infections (94.7\%), alopecia (89.5\%), severe infections $(10.5 \%)$, menstrual irregularities (33.3\%), and hematologic disturbances $(26.3 \%)$.
Conclusions A modified regimen of 9 monthly and 5 quarterly cyclophosphamide pulses may be an effective therapeutic option for childhood-onset LN.

\section{SYSTEMIC LUPUS ERYTHEMATOSUS AND ANTIPHOSPHOLIPID ANTIBODIES IN A CHILD WITH NOONAN SYNDROME}

${ }^{1}$ A Gupta*, ${ }^{1}$ A Kumar, ${ }^{2} \mathrm{~N}$ Todurkar, ${ }^{1} \mathrm{D}$ Suri, ${ }^{3}$ I Panigrahi, ${ }^{4}$ Ahluwalia, ${ }^{1} \mathrm{~S}$ Singh. ${ }^{1}$ PGIMER, Allergy Immunology Unit- Advanced Paediatrics Centre, Chandigarh, India; ${ }^{2} P G I M E R$, Advanced Paediatrics Centre, Chandigarh, India; ${ }^{3} P G I M E R$, Genetics and Metabolic UnitAdvanced Paediatrics Centre, Chandigarh, India; ${ }^{4}$ PGIMER, Department of Haematology, Chandigarh, India

\subsection{6/lupus-2017-000215.132}

Background and Aims Autoimmune dysfunction has been described in patients with Noonan Syndrome (NS). Till date, 9 patients with systemic lupus erythematosus (SLE) and NS have been reported. However, exact relationship between SLE and NS is not known.

Methods To describe the clinical presentation of a child with SLE and NS.

Results A 10-years-old boy presented with fever, gradually progressive dyspnoea, pain in bilateral elbow and knee joints for 8 months. His past and family history was normal. Weight and height were $<-3 \mathrm{z}$-score for his age. He had a long face with relatively large ears, down slanting palpebral fissure and livedo reticularis over lower limbs. Grade III ejection systolic murmur was present at pulmonary area along with loud second heart sound. He had hypertonia with brisk deep tendon reflexes in bilateral lower limbs, bilateral ankle clonus and extensor plantar response.

Investigations revealed anaemia, thrombocytopenia, leucopenia, elevated acute phase reactants and aPTT prolongation. His antinuclear antibody was positive by indirect immunofluorescence (4+ homogenous), anti-double stranded deoxyribonucleic acid was elevated and direct coomb test and lupus anticoagulant were positive along with low serum complement 3 level. Echocardiography revealed mitral valve prolapse and mitral regurgitation. Magnetic resonance imaging of brain revealed chronic lacunar infarct in medial aspect of right thalamus.

A diagnosis of SLE, APLA and NS was made and treatment with oral prednisolone, hydroxychloroquine, acetyl salicylate and low molecular weight heparin was initiated.

Conclusions Autoimmune conditions including SLE are being increasingly described in patients with NS requiring close monitoring and long term follow up.

\section{SOLUBLE CXCL16 IN JUVENILE SYSTEMIC LUPUS ERYTHEMATOSUS}

A Hassan. Tanta, Egypt

\subsection{6/lupus-2017-000215.133}

Background and Aims Systemic lupus erythematosus (SLE) is characterised by autoantibodies directed against self antigens, leading to inflammatory damage of many target organs. The infammatory soluble chemokine CXC motif ligand 16 sCXCL160) has been proposed as an important pathogenic mediator in inflammatory diseases, such as juvenileSLE. 